(C) 1993 IEEE. Personal use of this material is permitted. However, permission to reprint/republish this material for advertising or promotional purposes or for creating new collective works for resale or redistribution to servers or lists, or to reuse any copyrighted component of this work in other works must be obtained from the IEEE.

\title{
Simulation Support for Commissioning and Operating the SSC Linac*
}

\author{
F.W. Guy, J.W. Hurd, C.R. Chang, D.Raparia, C.Y.Yao \\ Superconducting Super Collider Laboratory \\ 2550 Beckleymeade Avenue, Dallas, Texas 75237
}

\section{Abstract}

Many different beam dynamics simulation techniques and codes will be used during commissioning of the SSC Linac. As commissioning progresses, these techniques and codes will be developed, improved, tested and integrated into the linac control system. When the initial commissioning is finished, the control system will include a solid basis of simulation capability for normal operational use and periodic tuneup. For instance, some of the procedures that must be supported during both commissioning and normal operation are matching, steering, acceptance scanning, RF tuneup and emittance measurements. These all require simulation capability that should be easily available to the operator in a form that: 1) takes its input directly from linac diagnostics; 2) is immediately useful without sophisticated analysis; and 3) produces whatever output is best suited for the task at hand, be it a graphic on a screen or a control signal to an actuator. We discuss the various approaches that are being pursued to ensure that the necessary beam dynamics simulation capability will be available as needed.

\section{INTRODUCTION}

Beam dynamics simulation of the SSC Linac can be divided into two major categories. These are on-line and offline simulation, with some overlap between them. On-line simulation uses capabilities built into the accelerator control system. Its main purpose is to monitor, test and adjust the accelerator during actual operation. Off-line simulation, using computers separate from the control system, has several purposes. It is used for design, parameter and error studies. Another important function is to generate performance predictions that can be compared with experimental measurements to verify both the beam dynamics codes and the accelerator models that have been used in those codes. Offline simulation is also an essential tool to track down and correct errors in design, manufacture, installation, adjustment and operation, and to generate simulated "data" to develop and check on-line simulation capability.

No beam-dynamics code can do a good job unless its model of the accelerator is reasonably accurate. Our models start with the SSC Linac design configuration, are modified within or outside the specified tolerances for error studies, and will be corrected by measurements of the actual as-manufactured assemblies as these measurements become available. There are many beam dynamics codes available of widely varying complexity, physics capability, user-friendliness and

\footnotetext{
* Operated by the University Research Association, Inc. for the U.S. Department of Energy, under contract No. DE-AC35-89ER40486.
}

computer requirements. Given limited time and resources, how does one choose the "best" beam dynamics code for a particular simulation need? An important criterion is familiarity. It takes time and practice to learn the correct use of complicated codes, to understand their physics and to know their limitations. The codes mentioned in this paper are those we presently anticipate using; we believe they will do the job and we have experience with them. Many other good codes are available to us and no doubt we will add some of these to our arsenal as we perceive the requirements.

\section{BEAM DYNAMICS SIMULATION CODES FOR ON-AND OFF-LINE ANALYSIS}

\section{A. Matrix Codes}

These codes are fast and easy to use, but usually cannot calculate second-order effects or those due to non-uniform, non-elliptical or non-symmetric beam charge distributions. The well-known user-friendly matrix optics codes TRACE2D and TRACE3D [1] are used for preliminary design, for instance to set up beamline geometry in matching sections or LEBTs. They provide quick calculations of focusing, matching, phase advances, beam parameters, etc. TRACE physics is used in two specialized codes, PARTRACE [2] and CCLTRACE [3], used for error studies in, respectively, the DTL (Drift-Tube Linac) and the CCL (Coupled-Cavity Linac). TRACE has proven quite useful in our work. We plan to integrate the TRACE physics and user interface into many of our on-line simulation and control functions.

\section{B. Ray-tracing Codes}

AXCEL [4] has been used for design and analysis of the einzel lens LEBT (Low-Energy Beam Transport) between the ion source and the RFQ (Radio Frequency Quadrupole). The code has axial symmetry and a limited set of input beam parameters. It calculates space-charge effect.

\section{C. "Particle-pushing" codes}

SSCL is using several codes in the PARMTEQ [5]PARMILA [6] family. These codes transport particles through elements of a beamline, such as RFQ or DTL cells. A similar code, CCLDYN [7], is used to simulate CCL beam dynamics. Most of these codes have been modified in one way or another to model unique features of the SSC Linac. Other particletransport codes used at SSCL for certain special problems are HESQT [8], BUCKSHOT [9], and ARGUS [10]. Multiparticle codes usually have long running times and are not suitable for integration into the on-line system except in a few special cases such as a single-particle version of 
PARMILA used for some steering and RF power setting algorithms.

\section{Beam Analysis Codes}

These are included here for completeness although they are not strictly simulation codes. We use two codes to analyze experimental data from the slit-and-collector beam emittance diagnostics. They are a code that resides in the TACL accelerator control system [11] and gives a preliminary on-line analysis of the collected data so that the operator may judge its quality; and REANE [12], an off-line analysis code with many special features. REANE has been modified by the SSC Linac Group to allow more flexibility in data analysis and to enable it to run on any UNIX platform with X11 X-Window capability. REANE input files generated by the TACL-based analysis code can be used to produce a file of particle coordinates for use in the PARMTEQ-_PARMILA codes; thus these beam analysis codes have become part of the simulation support effort.

\section{E. Codes for Setting RF Phase and Amplitude}

Accurate setting of RF power phase and amplitude in each tank is essential to maintain beam quality. Procedures for achieving this include the absorber-collector phase-scan, and the $\Delta \mathrm{t}$ [13] method. Both of these have proven very useful in other accelerators, and we will try a new method [14] called "least-squares" that calculates correct RF setting and actual input beam energy by minimizing the difference between measured and calculated beam output phase over a range of input beam energy and RF parameters. In preparing for commissioning, we are simulating each of these methods [15] off-line and intend to have them available in the control system. Phase scans and $\Delta t$ are inherently on-line methods. Least-squares requires more computation and may be done "semi-off-line" on a separate computer, but is reasonably fast.

\section{F. Special-Purpose Codes}

A number of small codes, both on- and off-line, will be available for purposes such as steering, emittance calculations from beam envelope measurements, and so forth.

\section{CODES FOR SPECIFIC LINAC SECTIONS}

\section{A. Ion Source}

$A b$-initio calculations of ion source output have not been used at SSCL because our sources are designed and built at other institutions. We have relied on beam measurements (which have certain complications and difficulties) or standard types of inputs such as waterbag, gaussian, etc. to provide the LEBT input in our calculations. SSCL has two types of $\mathrm{H}^{-}$ sources, magnetron and volume. The magnetron source [16] produces a divergent ion beam from a small aperture; the beam is fairly well characterized by a uniform angular distribution. The volume source [17] has a larger aperture and a more parallel beam. The distribution of ions across the aperture may not be uniform. There is a large electron component that must be removed by a spectrometer magnet a few centimeters from the aperture, which complicates the space-charge calculation and bends the ion beam slightly. The result is a non-uniform, non-symmetric beam. Eventually we expect to improve the extraction aperture and spectrometer design through beam-dynamics calculations, but for now we randomly sample the REANE input experimental data file to construct a file of input particle coordinates for the particlefollowing codes.

\section{B. Low Energy Beam Transport}

An einzel lens LEBT is presently being used in the SSC Linac and is producing a beam that is quite satisfactory for near-term goals [18]. The AXCEL code has been used for design and calculation of this lens. AXCEL has axial symmetry and therefore cannot accommodate input beams generated from experimental volume-source data, but it has been useful in showing how various symmetric beams respond to changes in electrode voltage. Transport of volume-source beams through the einzel lens could be calculated with a particlefollowing code such as BUCKSHOT or ARGUS, but because of time constraints this has not yet been done. The main purpose of LEBT beam-dynamics calculations is to provide the RFQ with a beam that will result in maximum transmission of accelerated beam. For the einzel lens, this goal can easily be realized during RFQ operation by trial-and-error adjustment of two high-voltage DC power supplies for the focusing electrodes, and four power supplies for steering; steering and focusing are weakly coupled as long as steering is small.

Two other candidates for the SSC LEBT are the Electrostatic Quadrupole (ESQ) [19] and Helical Electrostatic Quadrupole (HESQ) [20] geometries. These are more complicated than the einzel lens, but are expected to provide better quality beams to the RFQ. Bcam dynamics of these lenses can be calculated by TRACE, PARMILA, ARGUS and (for the HESQ) by DEEPRAP. Calculations are essential to determine proper steering and focusing voltages for these LEBTs because of the large number of power supplies; the HESQ, for instance, requires twelve DC power supplies, of which eight are used for both steering and focusing and four for focusing only. Optimizing the beam by trial-and-error would be a difficult (if not impossible) task; on-line simulation will be required.

\section{C. $R F Q$}

RFQ simulation at SSCL is handled almost entirely by variations of the PARMTEQ code and its associated support codes. The main exception is matching, done with TRACE. The code used at SSCL is adapted from an older Los Alamos National Laboratory version. SSCL's code was revised to include the higher-order multipole expansion of the vane tip field; the older LANL version had only the original "twoterm" field. This revision was done so that the SSCL version can accurately simulate the SSC RFQ, which was designed at LANL using a similar multipole treatment. These codes are based on a "z-dependent" treatment of the particle dynamics; there is also a "t-dependent" version of PARMTEQ that was 
furnished to SSCL by Chalk River Laboratories. The latter code also has a version of the multipole treatment. Both versions give approximately the same results on test problems.

Further extensions of RFQ simulation physics include 3-D space-charge and image charges. A version of PARMTEQ with these physics additions, including the multipole treatment, has been developed at SSCL. A previous 3-D space-charge, image-charge code has been reported in Reference [21]; 2-D vs. 3-D space-charge seems to make very little difference on any of the runs, but there are definite differences on some RFQs when image charges are used in the simulations. For the SSC RFQ, however, image charge effects are minimal.

\section{DTL and Matching Sections}

TRACE3D and PARMILA are the codes most used for beam-dynamics design and calculation of the drift-tube linac, the RFQ-DTL matching section, and the DTL-CCL matching section. Error studies generally use PARTRACE. Specialized versions of single- and multi-particle PARMILA are used in off-line steering, matching and RF power setting simulations; TRACE will be the principal physics package for on-line simulations of these sections. PARMILA has been modified to include focusing-magnet high-order multipoles.

Phase-scan will be used for setting the RF phase and amplitude in the matching section bunchers. Phase-scan on bunchers requires no simulation capability because the $90^{\circ}$ phase point and voltage can be determined directly from beam phase measurements. This technique is also good for preliminary RF adjustment on DTL tanks but requires longitudinal beam-dynamics simulation. This has been done for the SSC DTL. Results indicate that (assuming an accurate model) phase-scan can get "in the ballpark" but a more sophisticated technique is required for final adjustment of RF power. The $\Delta t$ method is one such; simulation capability for $\Delta \mathrm{t}$ will be provided in the control system. $\Delta \mathrm{t}$ will not work on the first DTL tank, because this method requires measurement of the beam throught the tank with the RF off, and the beam not only debunches but goes unstable. The least-squares method has been tested repeatedly with simulated noisy "data" and has worked very well with all SSC DTL tanks. This method provides a direct calculation of measurement errors in RF phase and amplitude and in input beam energy.

\section{E. $C C L$}

For the coupled-cavity linac, we use TRACE3D, CCLDYN and CCLTRACE. Steering and matching off-line simulations will be done with both TRACE and single- and multi-particle CCLDYN, error studies mostly with CCLTRACE, and on-line simulations by TRACE. The $\Delta t$ method will be used for RF settings. The least-squares method will probably be used as well; however, it has not yet been simulated for the CCL. CCLDYN has been modified to include focusing-magnet high-order multipoles and the "Yurij Effect" [22].

\section{CONCLUSIONS}

A variety of beam-dynamics simulation codes (and the expertise for their use) are available at the SSCL. Some codes will need to be modified and others created to satisfy the particular requirements of the SSCL. Altogether, we expect to have quite effective simulation capability and support for commissioning and operation of the SSC Linac.

\section{REFERENCES}

[1] K.R.Crandall, "TRACE, an Interactive Beam Dynamics Program," AIP Conference Proceedings 177, Linear Accelerator and Beam Optics Codes, La Jolla Institute, pp. 29-36 (1988).

[2] K.R. Crandall, "Error Studies Using PARTRACE, A New Program that Combines PARMILA and TRACE 3D," Proc 1988 Linear Accelerator Conference, pp. 335-337.

[3] CCLTRACE, K.R. Crandall, private communication.

[4] E.F. Jaeger and I.C. Whitson, "Numerical Simulation for Axially Symmetric Beamlets in the Duopigatron Ion Source," ORNL/TM-4990, Oak Ridge, TN (1975).

[5] K.R. Crandall, "PARMTEQ-A Beam-Dynamics Code for the RFQ Linear Accelerator," AIP Conference Proceedings 177, Linear Accelerator and Beam Optics Codes, La Jolla Institute, pp. 22-28 (1988).

[6] G.P. Boicourt, "PARMILA An Overview," ibid., pp 1-21: Los Alamos National Laboratory Report LA-UR-88-1544.

[7] CCLDYN, K.R. Crandall, private communication.

[8] HESQT, D. Raparia, private communication.

[9] J.S. Wagner, "BUCKSHOT, A 3-D Gridless Magnetostatic Particle Code," Sandia National Laboratory Report SAND87. 2019.

[10] ARGUS Code Manual, Science Applications International Corporation (1991).

[11] J. Sage, private communication.

[12) H.D. Holt, "Analyzing GTA Emittance Data," Los Alamos National Laboratory AT-10 Technical Note 91-01 (1991).

[13] K.R. Crandall, R.A. Jameson, D. Morris, and D.A. Swenson, "The $\Delta t$ Turn-On Procedure," Proc. 1972 Proton Linear Accelerator Conf., Los Alamos, NM, Oct 10-13, 1972, Los Alamos Scientific Report LA-5115, Nov. 1972, pp 122-125.

[14] F.W. Guy and T.P. Wangler, "Least-Squares Fitting Procedure for Setting RF Phase and Amplitude in Drift-Tube-Linac Tanks," Proc. 1991 IEEE Particle Accelerator Conference, Vol. 5, pp. 3056-3058.

[15] F.W. Guy and J.W. Hurd, "Procedures for Setting RF Phase and Amplitude in SSC Drift-Tube-Linac Tanks," Proc. 1992 Linear Accelerator Conference, Vol. 1, pp. 196-198.

[16] K. Saadatmand et. al., "Performance of the SSC. Magnetron Ion Source and LEBTs," ibid., pp. 94-96.

[17] K. Saadatmand and N.C. Okay, "Performance of the SSC RFDriven Volume Source," ibid., pp. 97-99.

[18] J. Lenz et. al., "Comparison of Experimental and Simulated Results for the SSC LEBT," these proceedings.

[19] S. Guharay et.al., "An ESQ Lens System for Low Energy Beam Transport Experiments on the SSC Test Stand," ibid.

[20] D. Raparia, "Beam Dynamics of the Low Energy Beam Transport and Radio Frequency Quadrupole," Ph.D. Thesis, University of Houston, Houston, TX (1990).

[21] F.W. Guy, "Three-Dimensional Space Charge and Image Charge Effects in Radio-Frequency-Quadrupole Accelerators," Proc. 1991 IEEE Particle Accelerator Conference, Vol. 5, pp. 3032-3034.

[22] Yu. Senichev et. al., "Beam Loading Effect in SSCL Coupled Cavity Linac," these proceedings. 